\title{
ESSAY
}

\section{Battle of the sexes may set the brain}

\section{A tug-of-war between the mother's and father's genes in the developing brain could explain a spectrum of mental disorders from autism to schizophrenia, suggest Christopher Badcock and Bernard Crespi.}

It has long been recognized that mental illnesses such as schizophrenia and autism tend to run in families. But neither disorder obeys classical Mendelian laws of inheritance, making it difficult to pinpoint the genes involved.

We believe that psychiatric illness may be less to do with the genes a mother and father pass down, and more to do with which genes they program for expression. By our hypothesis, a hidden battle of the sexes - where a mother's egg and a father's sperm engage in an evolutionary struggle to turn gene expression up or down - could play a crucial part in determining the balance or imbalance of an offspring's brain. If this proves true, it would greatly clarify the diagnosis of mental disorders. It might even make it possible to reset the mind's balance with targeted drugs.

The story begins with the late William Hamilton, originator of the selfish gene idea popularized by Richard Dawkins. Hamilton explained how genetic traits that are apparently injurious can evolve by natural selection. He proved that genes predisposing an individual to self-sacrifice could propagate in a population if they were sufficiently shared by the beneficiaries of such acts. Charles Darwin did not know about genes, but saw evolution in terms of conflict between individuals, groups and species. Hamilton showed that evolution is, in fact, a question of conflict between genes. Because individuals in sexually reproducing species receive genes from two different parents, he also realized that such conflicts probably occur within the same individual.

Shortly before Hamilton's untimely death in 2000 , evidence was found for this internal selfish-gene behaviour. Geneticists discovered that some crucial genes are expressed when inherited from one parent, but not when inherited from the other. This is achieved through a process called imprinting, in which genes in the sperm and egg are marked for expression or silencing in a later embryo and child. For example, a fetus inherits a gene called IGF2, which encodes an insulin-like growth factor, from both its mother and its father. In mammals, only the father's copy is normally expressed. In humans, if the mother's copy is also expressed, the result is a child with Beckwith-Wiedemann syndrome. This is characterized by a birthweight that is more than $50 \%$ above normal, with various other symptoms of over-growth. If, by contrast, both the father's and the mother's copies are silenced, the opposite outcome occurs - under-growth, as featured in Silver-Russell syndrome.

Larger babies live longer, develop less disease and have better all-round health. It is now thought that the genetic conflict over offspring size arises because the father's genes gain these benefits by being carried in a large offspring at no personal cost to the father. The mother, however, pays the costs of gestating, giving birth to and suckling a larger child. Hence, mammalian mothers silence their copies of growth-enhancing genes such as IGF2, whereas fathers mark them for expression.

Genes that are either maternally or paternally biased thus engage in a genetic tug-ofwar. Letting the rope slip in one direction or the other leads to opposite outcomes in offspring.

\section{Powerful pull}

It is currently thought that perhaps a few hundred of the 20,000 or so human genes are subject to imprinting, although only 63 are confirmed so far. This number might sound small, but imprinted genes such as IGF2 commonly have far-reaching effects on growth and development. Imprinting has been found to be especially frequent in genes expressed in the placenta, the organ that governs how resources are extracted from the mother. Imprinting is also common among genes that drive brain development. A reasonable conclusion is that genetic tugs-ofwar should also affect behaviour, cognition and personality.

Hamilton certainly thought that genetic conflict would have psychological consequences. He noticed that there were 'people people' and 'things people', classifying himself as the latter. Although these tendencies could be "disastrous socially", he wrote, "I believe it is in essence an aberration of this kind that makes me a successful scientist"1. When taken to an extreme, the social difficulties and mechanistic tendencies of 'things people' are recognizable as symptoms of autism.

Autistic children are notably self-oriented and demanding on their care-givers; throughout evolutionary history, this has usually been the mother. This led us to suggest in 2006 that some cases of autism may be the result of paternally biased expression of genes involved with brain development ${ }^{2}$. Since then, investigations of patients with Beckwith-Wiedemann syndrome have revealed that they have a greatly increased risk of autism, and people with autism tend to have enhanced expression of IGF2. These associations support a link between autism and imprinting.

Although there is clearly a wide range of risk factors for autism, including some known toxins, genetic and hormonal factors, the vast majority of cases remain of unknown cause. Imprinting could be behind such instances.

If imprinting in one direction does indeed cause autism, then imprinting in the other direction should have some opposite effect. It struck one of us (C.B.) that some of the fundamental deficits of autism described in Simon Baron-Cohen's 1995 book Mindblindness contrast with classic symptoms of paranoia. For example, the defective detection of gaze seen in autism seems to be the exact opposite of paranoid delusions of being watched or spied on. The autistic inability to appreciate what goes on in groups can also be seen as the antithesis of paranoid delusions of conspiracy, which involves imagining group activity everywhere. Furthermore, the religious, magical and mystical delusions that cause people with paranoia to see evidence of mind, intention and meaning in everything seem to be the opposite of autistic deficits in theory of mind, which result in an inability to understand that others have their own beliefs and intents.

We have become convinced that autism can be considered as the diametric opposite of both paranoia and the full spectrum of related psychotic and mood disorders, which include schizophrenia, bipolar disorder and major depression. Numerous other antithetical symptoms fit this broader contrast: autistic single-mindedness versus psychotic ambivalence, autistic candour versus psychotic selfdeception, autistic deficits in a sense of self versus psychotic megalomania and delusions of grandeur, and so on.

In summary, we propose that autistic spectrum conditions are characterized by deficits in theory-of-mind skills, or 'hypo-mentalism', 


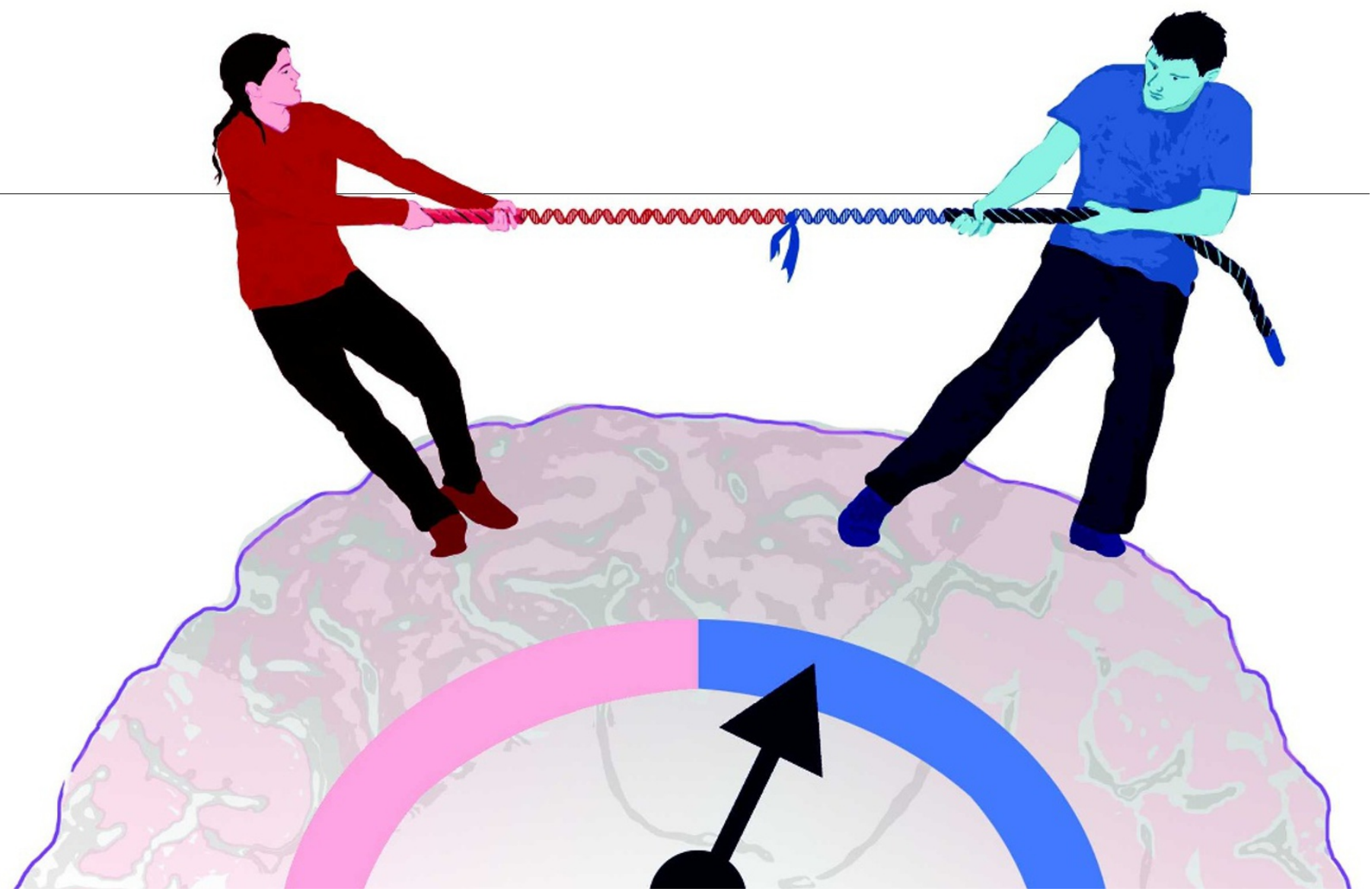

whereas psychotic spectrum conditions involve the exact opposite: 'hyper-mentalism'.

According to this theory ${ }^{3}$, small deviations in imprinted-gene expression towards a maternal bias should result in smaller babies that are energetically 'cheaper' to mothers, and who are easier behaviourally - more placid, less demanding and more mentalistically attuned to interpreting and understanding the mental states of others. Large maternally biased deviations should lead to psychosis. Conversely, small alterations towards paternal bias should lead to relatively demanding children who are more focused on 'things'; larger paternal biases should cause the severe hypo-mentalistic deficits of autism. Between these extremes would sit normal cognition.

To propose a single, overriding explanation for such a huge range of mental conditions is controversial. But there seems to be some evidence that fits, from genetics as well as epidemiology.

\section{Evidence accrues}

Geneticists have found a region of human chromosome 15, for example, that contains a set of imprinted genes. Children with a paternal bias in gene expression in this area develop Angelman or 'happy puppet' syndrome, which involves hyperactive, attention-seeking behaviour in infancy and a very high incidence of autism. Children with a maternal bias in this area develop PraderWilli syndrome, which features extremely placid, undemanding behaviour in infancy, followed after weaning by compulsive food- seeking, which can be seen to reduce the demands on a mother. This is accompanied by rates of psychosis with depression that can approach $100 \%$.

The theory also fits neatly with another notable contrast between autistic and psychotic spectrum conditions: age of onset. Autistic disorders typically become noticeable in childhood; psychosis mostly develops in late adolescence or early adulthood. This may be because theory-of-mind skills take many years to master, so under-development of these skills is noticed much earlier than over-development.

The gene-expression tug-of-war could also help to explain sex biases in the prevalence and severity of different mental illnesses. Recent studies by Baron-Cohen have persuasively linked autistic tendencies with exposure of the fetus to testosterone, partly explaining the considerable male bias in vulnerability to autism. But Baron-Cohen's 'extreme male brain' theory does not predict the fact that autism, although rarer in girls, is generally more severe in females. Conversely, depression is more common in females, and schizophrenia is more often severe among males.

According to our theory, there are two major axes of cognition: one determined by sex, and one by the paternal or maternal bias in gene expression. Perhaps mental disorders are more common but less severe where these two axes are compatible: in males with autism and females with depression. When the two axes are least well-matched, as in females with autism and males with psychosis, disorders seem to be much more severe.

The 'imprinted brain' hypothesis could be probed through an extensive research programme spanning studies of genes, neurodevelopment, social cognition and mental conditions. It is vital to establish a full list of the genes imprinted in the human brain, determine what these genes do, and discover how variation in their expression contributes to the development of autistic versus psychotic spectrum conditions. The search for imprinted genes is under way, and methods for finding them are improving. Still, not enough attention is focused in this direction.

Our theory of genomic conflict in brain development represents the first conceptual bridge, grounded in biology, that spans the major disorders of the social brain. If our theory proves correct, Hamilton's essential insight into genetic conflict as the fundamental driver of evolution would be vindicated, and the intricate underpinnings of mental conditions such as psychosis, or his own apparent mild autism, would be much better understood. Christopher Badcock is a reader in sociology at the London School of Economics, Houghton Street, London WC2A 2AE, UK.

Bernard Crespi is professor of evolutionary biology at Simon Fraser University, Burnaby, British Columbia V5A 1S6, Canada.

e-mail: crespi@sfu.ca

1. Hamilton, W. D. in Narrow Roads of Gene Land Vol.3: Last Words. 206 (W. H. Freeman/Spektrum, 2005).

2. Badcock, C. \& Crespi, B. J. Evol. Biol. 19, 1007-1032 (2006).

3. Crespi, B. \& Badcock, C. Behav. Brain Sci. 31, 241-261 (2008). 